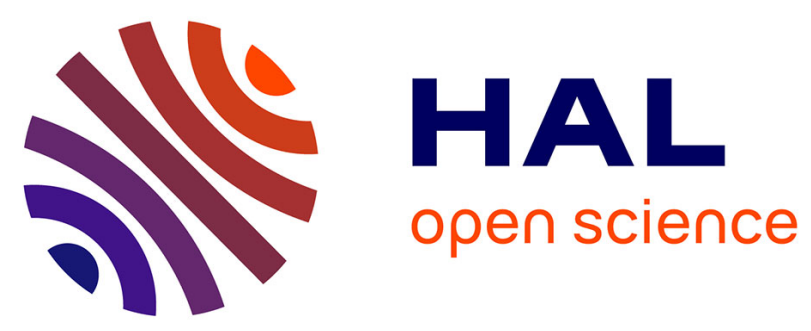

\title{
Distinct phospholipid and sphingolipid species are linked to altered HDL function in apolipoprotein A-I deficiency
}

Emile Zakiev, Fabiana Rached, Marie Lhomme, Maryam Darabi-Amin, Maharajah Ponnaiah, Pierre Hadrien Becker, Patrice Therond, Carlos Serrano, Raul Santos, M. John Chapman, et al.

\section{To cite this version:}

Emile Zakiev, Fabiana Rached, Marie Lhomme, Maryam Darabi-Amin, Maharajah Ponnaiah, et al.. Distinct phospholipid and sphingolipid species are linked to altered HDL function in apolipoprotein A-I deficiency. Journal of clinical lipidology, 2019, 13 (3), pp.468-480.e8. 10.1016/j.jacl.2019.02.004 . hal-02166947

\section{HAL Id: hal-02166947 \\ https://hal.sorbonne-universite.fr/hal-02166947}

Submitted on 25 Oct 2021

HAL is a multi-disciplinary open access archive for the deposit and dissemination of scientific research documents, whether they are published or not. The documents may come from teaching and research institutions in France or abroad, or from public or private research centers.
L'archive ouverte pluridisciplinaire HAL, est destinée au dépôt et à la diffusion de documents scientifiques de niveau recherche, publiés ou non, émanant des établissements d'enseignement et de recherche français ou étrangers, des laboratoires publics ou privés.

\section{(c) $(1)$}

Distributed under a Creative Commons Attribution - NonCommerciall 4.0 International 
Distinct phospho- and sphingolipid species are linked to altered HDL function in apolipoprotein A-I deficiency

Emile Zakiev MSc ${ }^{a}$, Fabiana Rached MD, $\mathrm{PhD}^{\mathrm{b}}$, Marie Lhomme $\mathrm{PhD}^{\mathrm{c}}$, Maryam Darabi-Amin $\mathrm{PhD}^{\text {a }}$, Maharajah Ponnaiah $\mathrm{PhD}^{\mathrm{c}}$, Pierre Hadrien Becker, $\mathrm{PhD}{ }^{\mathrm{d}}$,Patrice Therond MD, PhD ${ }^{\mathrm{d}}$, Carlos V. Serrano Jr. MD, PhD ${ }^{\mathrm{b}}$, Raul

D. Santos MD, $\mathrm{PhD}{ }^{\text {be }}$, M. John Chapman $\mathrm{PhD}^{\mathrm{a}}$, Alexander Orekhov $\mathrm{PhD}{ }^{\mathrm{fg}}$, Anatol Kontush $\mathrm{PhD}{ }^{\mathrm{a}}$

Running Title: ApoA-I deficiency and dysfunction of HDL

${ }^{a}$ UMR-ICAN 1166, National Institute for Health and Medical Research (INSERM), Sorbonne University, Paris, France

${ }^{\mathrm{b}}$ Heart Institute (InCor), University of Sao Paulo Medical School Hospital, Sao Paulo, Brazil

${ }^{c}$ ICANalytics, Institute of Cardiometabolism and Nutrition (IHU-ICAN, ANR-10-IAHU-05), Paris, France

${ }^{d}$ Biochemistry Laboratory of Bicêtre Hospital, Le Kremlin-Bicêtre, France

${ }^{\mathrm{e}}$ Hospital Israelita Albert Einstein, Sao Paulo, Brazil

${ }^{\mathrm{f}}$ Institute of General Pathology and Pathophysiology, Moscow, Russia

${ }^{\mathrm{g}}$ Institute for Atherosclerosis Research, Moscow, Russia

Corresponding author: Dr. Anatol Kontush, INSERM UMR_S 1166 - ICAN, Faculté de Médecine Pitié-

Salpêtrière, Sorbonne University, 91, Bd de l'Hôpital, 75013 Paris, France. Tel. (33) (1) 407796 33. Fax (33) (1)

407796 45. E-mail anatol.kontush@upmc.fr 
Background: Familial apolipoprotein A-I (apoA-I) deficiency (FAID) involving low levels of both apoA-I and high-density lipoprotein (HDL)-cholesterol is associated with accelerated atherosclerosis.

Objective: To define distinctive patterns in the lipidome of HDL subpopulations in FAID in relationship to antiatherogenic activities.

Methods: Five HDL subfractions were isolated by ultracentrifugation from plasma of FAID Caucasian patients $(\mathrm{n}=5)$ and age-matched healthy normolipidemic Caucasian controls $(\mathrm{n}=8)$, and the HDL lipidome (160 molecular species of 9 classes of phospho- and sphingolipids) was quantitatively evaluated.

Results: Increased concentrations of numerous molecular species of lysophosphatidylcholine (up to 12-fold), ceramides (up to 3-fold), phosphatidylserine (up to 34-fold), phosphatidic acid (up to 71-fold) and phosphatidylglycerol (up to 20-fold) were detected throughout all five HDL subpopulations as compared to their counterparts from controls, whereas concentrations of phosphatidylethanolamine species were decreased (up to 5fold). Moderately- to highly-abundant, within their lipid class, species of phosphatidylcholine, sphingomyelin, phosphatidylinositol, phosphatidylethanolamine, phosphatidylserine and ceramide featuring multiple unsaturations were primarily affected by apoA-I deficiency; their HDL content, particularily that of phosphatidylcholine(34:2), was strongly correlated with HDL function, impaired in FAID. Metabolic pathway analysis revealed that sphingolipid, glycerophospholipid, and linoleic acid metabolism was significantly affected by FAID.

Conclusion: These data reveal that altered content of specific phospho- and sphingolipid species is linked to deficient anti-atherogenic properties of HDL in FAID.

Keywords:

apoA-I-deficiency; lipidomics; familial dyslipidemia; HDL; Apolipoproteins 
Hypoalphalipoproteinemia, equally termed genetic HDL deficiency, accounts for around $4 \%$ of all severe premature cardiovascular disease (CVD) cases [1, 2]. Apolipoprotein A-I (apo A-I) is a major protein of highdensity lipoprotein (HDL), comprising around 35\% of total HDL mass and 70\% of HDL protein [3]. Homozygous subjects with genetic HDL deficiency resulting from impaired apoA-I production typically display extremely low HDL-cholesterol (HDL-C) levels of less than $5 \mathrm{mg} / \mathrm{dl}$ [4]. Familial ApoA-I deficiency can be manifested alone or in association with apoCIII/apoA-IV or apoC-III deficiency. ApoA-I plays a key role in cellular cholesterol efflux and displays other atheroprotective activities, including antioxidative, anti-inflammatory, anti-thrombotic and antiapoptotic actions [5]. Moreover, apoA-I occupies central place in lipoprotein metabolism, reflecting a fine balance between its lipid-associated and not lipid-associated states [6]. As a consequence, HDL metabolism, structure, function and composition are markedly affected in familial ApoA-I deficiency.

A large body of evidence documents a role for genetic regulation of the composition (lipidome, proteome) and biological function of HDL [7-11]. In our previous studies [12], we observed that HDL subpopulations isolated from patients with nonsense mutation at codon 2, Q[-2]X in the APOA1 gene, first described by Santos et al [13], possessed altered chemical composition. In particular, apoA-I, phospholipid, and cholesteryl ester contents were decreased while those of apoA-II, free cholesterol, and triglycerides (TG) were increased relative to HDL from healthy normolipidemic controls; furthermore, several lipid classes of the HDL phosphosphingolipidome were affected. In parallel, both small, dense HDL3 subfractions and total HDL exhibited defective antiatherogenic activity when compared to controls. Remarkably, cholesterol efflux capacity and antioxidative function of HDL subpopulations, isolated from patients, were reduced by up to $25 \%$ of their normal values. The diminished atheroprotective properties of HDL were correlated with altered lipid and apolipoprotein composition.

In continuation of these studies, we aimed to characterize lipidomic alterations in apoA-I-deficient HDLs at the molecular level in order to clarify which lipid species might contribute to functional HDL deficiency in this clinical condition. Our present in-depth analysis reveals that the most prominent alterations were observed in 
moderately to highly abundant, in their respective lipid classes, phospho- and sphingolipid species possessing multiple unsaturations in their fatty acid residues. Importantly, altered HDL content of these species, essentially, was tightly linked to deficient anti-atherosclerotic activities of HDL, potentially reflecting modulation of several genes involved in lipid metabolism in apoA-I deficiency. 


\section{Materials and Methods}

\subsection{Subjects}

We studied four Caucaisan male and one female heterozygous patients from a family with a nonsense mutation at APOA1 codon -2, Q[-2]X, genotyped according to Santos et al [13], and eight healthy male Caucasian normolipidemic, non-smoking age-matched controls with no signs of a known disease, all recruited at the Heart Institute (InCor) of the University of Sao Paulo Hospital in Sao Paulo, Brazil, as previously described [12]. None of the patients or controls had pathologies affecting lipid metabolism, including Type 2 diabetes, kidney disease and thyroid disease. All subjects were off lipid-lowering medication for at least 2 months at the time of assessment. As we reported earlier [12], heterozygous apoA-I-deficient patients featured atherogenic dyslipidemia with significantly reduced apoA-I and HDL-C levels, while no difference between the groups in TG, total cholesterol, low-density lipoprotein cholesterol, apoB-100, apoC-III, fasting glucose and hsCRP levels was observed (Supplementary Table S1) [12]. Written informed consent was obtained from all subjects, and the project was approved by the Ethics Committee of the Heart Institute-InCor in accordance with local institutional guidelines conformed to the Declaration of Helsinki.

\subsection{Blood samples and isolation of lipoproteins}

Blood samples were drawn from the cubital vein of each participant after overnight fasting and were centrifuged at low speed at $4{ }^{\circ} \mathrm{C}$ yielding EDTA plasma and serum, which were stored at $-80^{\circ} \mathrm{C}$ as described elsewhere [12]. An aliquot of $3 \mathrm{ml}$ serum was thawed and subjected to a single step, isopycnic nondenaturing density gradient ultracentrifugation in a Beckman SW41 Ti rotor at 40,000 rpm for $44 \mathrm{~h}$ in a Beckman XL70 ultracentrifuge at $15^{\circ} \mathrm{C}$ as described previously $[14,15]$. After the ultracentrifugation, five major HDL subfractions (HDL2b, 2a, 3a, $3 b$ and $3 c)$ were isolated and used for lipidomic analysis and functional studies. As small dense HDL3b and 3c particles display more potent biological activities as compared to large, light HDL2 [10, 16] and are markedly affected in apoA-I deficiency [12], data on HDL3b and 3c are included in the main manuscript, while those on the other HDL subpopulations are placed in the Supplementary Materials. 


\subsection{Compositional analyses}

Molecular isobaric lipid species $(\mathrm{n}=160)$ belonging to seven principal glycerophospate subclasses

[phosphatidylcholine (PC), lysophosphatidylcholine (LPC), phosphatidylethanolamine (PE), phosphatidylinositol (PI), phosphatidylglycerol (PG), phosphatidylserine (PS), and phosphatidic acid (PA)] and two principal sphingolipid subclasses [sphingomyelin (SM) and ceramide (Cer)], were quantitatively assayed by LC/MS/MS as described elsewhere [12]. Extraction, LC/MS analysis and quantification were performed according to [12]. A blank (PBS) and a control (HDL2 obtained from a reference normolipidemic plasma) sample were extracted in parallel with each batch to ensure quality control; each sample was corrected for blank readings, as described in [12]. Sphingosine-1-phosphate (S1P) content of HDL was assessed using HPLC, as described elsewhere [17].

Lipid standards used for quantification of HDL lipidome were as described elsewhere [12]. 1,2-Dipalmitoyl-snglycero-3-phosphocholine-N,N,N-trimethyl-d9 (PC 16:0/16:0 d9), 1-lauroyl-2-tridecanoyl-sn-glycero-3-phospho(1'-myo-inositol) (PI 12:0/13:0), 1-dodecanoyl-2-tridecanoyl-sn-glycero-3-phosphoethanolamine (PE 12:0/13:0), 1-dodecanoyl-2-tridecanoyl-sn-glycero-3-phospho-(1'-rac-glycerol) (PG 12:0/13:0), 1-dodecanoyl-2-tridecanoylsn-glycero-3-phosphate (PA 12:0/13:0), 1-dodecanoyl-2-tridecanoyl-sn-glycero-3-phospho-1-serine (PS 12:0/13:0), 1-pentadecanoyl-2-hydroxy-sn-glycero-3-phosphocholine (LPC 15:0), and N-heptadecanoyl-derythro-sphingosine (Cer d18:1/17:0) were used as internal standards. 1-Palmitoyl-2-hydroxy-sn-glycero-3phosphocholine (LPC 16:0), 1-stearoyl-2-hydroxy-sn-glycero-3-phosphocholine (LPC 18:0), 1,2-dimyristoyl-snglycero-3-phosphocholine (PC 14:0/14:0), 1-myristoyl-2-palmitoyl-sn-glycero-3-phosphocholine (PC 14:0/16:0), 1,2-dipalmitoyl-sn-glycero-3-phosphocholine (PC 16:0/16:0), 1-palmitoyl-2-stearoyl-sn-glycero-3-

phosphocholine (PC 16:0/18:0), 1-palmitoyl-2-oleoyl-sn-glycero-3-phosphocholine (PC 16:0/18:1), 1-palmitoyl2-linoleoyl-sn-glycero-3-phosphocholine (PC 16:0/18:2), 1,2-distearoyl-sn-glycero-3-phosphocholine (PC 18:0/18:0), 1-stearoyl-2-oleoyl-sn-glycero-3-phosphocholine (PC 18:0/18:1), 1-stearoyl-2-linoleoyl-sn-glycero-3phosphocholine (PC 18:0/18:2), 1-stearoyl-2-arachidonoyl-sn-glycero-3-phosphocholine (PC 18:0/20:4), 1palmitoyl-2-docosahexaenoyl-sn-glycero-3-phosphocholine (PC 16:0/22:6), 1-stearoyl-2-docosahexaenoyl-snglycero-3-phosphocholine (PC 18:0/22:6), 1-stearoyl-2-hydroxy-sn-glycero-3-phosphoethanolamine (LPE 18:0), 1,2-distearoyl-sn-glycero-3-phosphoethanolamine (PE 18:0/18:0), 1-heptadecanoyl-2-(9Z-tetradecenoyl)-sn- 
glycero-3-phospho-(1'-myo-inositol) (PI 17:0/14:1), N-stearoyl-d-erythro-sphingosine (Cer d18:1/18:0), 1,2distearoyl-sn-glycero-3-phosphate (PA 18:0/18:0), 1,2-distearoyl-sn-glycero-3-phospho-(1'-rac-glycerol) (PG 18:0/18:0), and 1-palmitoyl-2-linoleoyl-sn-glycero-3-phospho-1-serine (PS 16:0/18:2) were purchased from Avanti Polar Lipids (Alabaster, AL) to construct separate calibration curves as a function of the number of double bonds in fatty acid moieties and of their chain length. Adequate lipid recovery across different samples was ensured by optimising extraction conditions ( $\mathrm{pH}$ and solvent v/v ratios) of the classical Folch extraction [18] to improve the recovery of negatively charged lipids. LC/MS-grade solvents were used without further purification and obtained from Sigma-Aldrich (St. Louis, MO) or VWR (West Chester, PA).

ApoA-I and apoA-II were measured in HDL by immunoturbidimetry [12].

\subsection{Functional assays: cholesterol efflux, antioxidative and anti-apoptotic activities of HDL}

Cholesterol efflux capacity of HDL subpopulations was assessed, as described elsewhere [12]. In short, THP-1 monocytes were cultured and differentiated into macrophage-like cells using PMA for $48 \mathrm{~h}$. The cells were then washed and loaded with $\left[{ }^{3} \mathrm{H}\right]$ cholesterol-labeled acLDL. Cellular cholesterol efflux to $15 \mu \mathrm{g} / \mathrm{ml} \mathrm{HDL}-\mathrm{GP}$ was assayed in serum-free medium for a $4 \mathrm{~h}$ chase period and quantified using liquid scintillation counter.

Antioxidative capacity of HDL3b and HDL3c subpopulations was assessed as described elsewhere [12]. In short, ability of $10 \mathrm{mg}$ total mass/dl of HDL to inhibit oxidation by azo-initiator 2,2'-azo-bis-(2-amidinopropane) hydrochloride in reference LDL isolated from one healthy normolipidemic control subject was measured as accumulation of conjugated dienes in absorbance at $234 \mathrm{~nm}$.

Anti-apoptotic activity of HDL was assayed as described elsewhere [19] with slight modifications. In brief, human umbilical vein endothelial cells (HUVEC) purchased from PromoCell (Germany) and maintained in Endothelial Cell Basal Medium (C-22111, Promocell) supplemented with fetal bovine serum (20\%, v/v) and human basic fibroblast growth factor. Growth factor deprivation was induced by washing the cells two times with phosphate buffered saline (PBS) and changing the media to Endothelial Cell Basal Medium without supplements. Deprived cells were either incubated with HDL at a final concentration of $100 \mu \mathrm{g}$ protein/ml or without HDL 
(substituted with PBS) for $16 \mathrm{~h}$. Floating endothelial cells were collected by centrifugation and adherent cells were collected by trypsinization. For determination of the annexin $V$ binding, combined cell populations $(5 \times$ 105/ml) were resuspended in $140 \mathrm{mM} \mathrm{NaCl}, 10 \mathrm{mM}$ Hepes buffer, and $2.5 \mathrm{mM} \mathrm{CaCl}_{2}$. Annexin V-FITC (BioLegend's FITC Annexin V Apoptosis Detection Kit with 7-AAD) was added for 15 min at room temperature. For determination of the cell membrane permeability, endothelial cells $\left(5 \times 10^{5} / \mathrm{ml}\right)$ were incubated for $30 \mathrm{~min}$ in PBS. Flow cytometric measurements of annexin V binding were performed on a BD LSRFortessa flow cytometer (BD Biosciences, Franklin Lakes, New Jersey, USA).

\subsection{Statistical analysis}

Between-group differences were analyzed using Mann-Wilcoxon U-test. Pearson's product moment correlations were calculated to evaluate relationships between variables. For all statistical tests, a p value of less than 0.05 (Benjamini-Hochberg adjusted, otherwise known as False Discovery Rate (FDR) corrected) was considered statistically significant.

Principal Component Analysis (PCA) was performed to provide a broad view on a dataset, emphasizing its most valuable informationwise subsets. PCA reduces intricate multiple-dimension data into several (usually two, less often three or more) most meaningful Principal Components in terms of information carried by them by maximizing the variance among the components. In this study Principal Components (eigenvectors and eigenvalues) were calculated using R built-in prcomp function with scale parameter set True; corresponding charts were built using ggbiplot $\mathrm{R}$ package.

Heatmap is a graphical representation of data where individual values contained in a matrix are represented as colors. To create heatmaps, following rules were employed: (i) abundance of each lipid species from the patient group was normalized to the mean value of the abundances of this species in the control group; (ii) the given species was represented as a single colored bar; (iii) the bar color corresponded to the ratio of the abundances of this species between the patient and the control groups, varying from red (decreased abundance in the patient vs. 
control group) through black (no difference) to green (increased abundance in the patient vs. control group). All the charts were built using R, specifically the heatmap. 2 function from gplots package.

Bubble plots were employed, which can be described as a more sophisticated variety of a heatmap with multiple additions, to provide a more in-depth view on the data as compared to heatmaps. As in the heatmaps, the main type of information conveyed in this plot type was the ratio of mean abundance of any given molecular species from the patient group to the mean abundances of the same species in the control group. In addition, the bubble plots provided quantitative information about mean abundances for all molecular species from the patient group. Finally, the bubble plots contained p-values of the differences between abundances of lipid species in the patient vs. control groups, thereby excluding from the plots all species which were not significantly different between the groups. Every molecular species was presented as a colored circle ("bubble"), the color coded for phospho- and sphingolipid class. The circle position along $\mathrm{X}$ axis represented the abundance of the species, and the position along the $\mathrm{Y}$ axis represented the ratio of the abundances in the patient vs control groups. The size of the bubble was inversely proportional to the $\mathrm{p}$ value mentioned above. All the axes as well as the size of the bubbles were plotted using logarithmic scale.

Structural 3D plots were built to convey information about the structure of fatty acid residues of affected lipid species, notably the number of double bonds and the length of carbon chains. Each dot represented a single molecular species, with a number of double bonds displayed along the $\mathrm{X}$ axis and a number of carbon atoms in the fatty acid chains displayed along the $\mathrm{Z}$ axis. The $\mathrm{Y}$ axis enlisted all five HDL subfractions. Only molecular species that revealed statistically significant differences between the patient and control groups were shown in the plots.

Network maps offer a graph-type visual representation of all intrinsic correlations within a dataset. Network maps were created in order to reveal relationships between the lipidome, proteome and function of HDL. In a given network community a network map reveals all interconnections between the nodes of this community. Assuming the HDL lipidome and function representing the community, molecular species and functional metrics stood for the nodes of the graph. If there was a significant correlation between abundances of any two species of the lipidome or functional metrics (nodes), these species and metrics were assumed to have a connection (edge). The 
length of the vertices between the nodes was proportional to the value of the correlation coefficient between the abundances of the lipid species represented by the nodes. Size of the node was proportional to the number of neighbouring vertices connected to the node. Color of the node indicated lipid class each species belonged to, as for the bubble plots described above. All lipid species from all HDL subpopulations were employed to build network maps. To correlate the lipidomic data with the functional metrics, the species abundances were recalculated on the HDL concentration basis employed to evaluate a given metric (phospholipid for cholesterol efflux capacity and total mass for antioxidative activity).

Affected metabolic pathways were identified and included in classical pathway representation after identification using the MetExplore web-based tool [20]. The metabolic network Homo sapiens (strain: Global Network, source: KEGG Map, Version 24/08/2017) of human species was based on 88 metabolic pathways including 1572 metabolites [21]. Metabolic networks are directed graphs, so it is possible to calculate compound importance based on relative betweenness centrality and out-degree centrality of any given compound from a pathway. Pathway impact then is calculated as a sum of the importance measures of the matched metabolites normalized by the sum of the importance measures of all metabolites in each pathway.

Affected genes were identified using Metaboanalyst's Network Explorer tool [22]. Metaboanalyst uses Globaltest [23] and GlobalANCOVA [24], which are similar algorithms designed for testing differentially expressed genes or metabolites in functionally related groups. Degree centrality and betweenness centrality are two measures to estimate the importance of a compound within a given gene-metabolic network and both are employed to find the affected genes. The former measures the number of connections which the node of interest has to other nodes, while the latter measures the number of shortest paths going through the node of interest. 


\section{Results}

\subsection{Abundances of lipid species}

In terms of their overall abundance, PC predominated in the HDL lipidome followed by SM, LPC, PI, PE, PS, Cer, PA and PG $[10,12]$. The HDL lipidome was markedly perturbed in apoA-I-deficient patients as reported by us earlier [12]. Among the nine phospho- and sphingolipid subclasses studied, PA and PG were most strongly affected. Indeed, total PA and PG were significantly increased in apoA-I-deficient patients relative to controls in all five HDL subpopulations, including both large, light and small, dense HDLs [12]. Other lipid classes showed significant between-group differences in three (PC decreased in HDL2b, HDL3b, HDL3c; LPC elevated in HDL2b, HDL3b and HDL3c; PI elevated in HDL2b, HDL3a and HDL3c; PS increased in HDL2b, HDL2a and HDL3c), two (SM elevated in HDL2b and decreased in HDL3a) and one (PE decreased in HDL2b; Cer elevated in HDL2b) subpopulations [12].

When abundances of lipid species were analysed, all HDL subpopulations revealed significant between-group differences. However, the number of significantly differing (FDR-corrected) between the groups species varied greatly across the HDL subpopulations for all lipid classes, including PC (from 1 to 6), SM (from 2 to 7), LPC (from 1 to 9), PI (from 1 to 3), PE (from 1 to 5), PS (from 2 to 8), Cer (from 1 to 19), PA (from 5 to 10) and PG (from 3 to 9) lipid species (Supplementary Tables S2 and S3). Significant between-group differences (FDRcorrected) were typically found in the abundances of unsaturated lipid species. Indeed, among molecular species significantly differing between apoA-I-deficient and control groups 92\% (55 of 61), 95\% (18 of 19), 94\% (16 of 17), $91 \%$ (30 of 33) and $93 \%$ ( 51 of 55) possessed one or more double bond in their fatty acid carbon chain residues in HDL2b, 2a, 3a, 3b and 3c, respectively.

\subsection{PCA}

When PCA was applied to our dataset, the control and patient groups were entirely separated for the lipidome of both small, dense (Fig. 1) and large, light (Supplementary Figure S1) HDL subpopulations on a plane of the first 
two Principal Components, indicating that individual components of the HDL lipidome were strongly altered in apoA-I-deficient subjects. Indeed, for all five HDL subpopulations, the amount of variance explained by the first two Principal Components was higher than 45\%, consistent with marked differences between the groups.

\subsection{Heatmaps}

When heatmaps were built for the differences in the abundances of lipid species between apoA-I-deficient and control subjects, a distinct pattern was observed across all HDL subpopulations (Fig. 2; Supplementary Figure S2). Notably, abundances of LPC, PA, PG and, to a lesser extent, Cer species were typically elevated in apoA-Ideficient HDLs as compared to their counterparts from controls, while the content of PE species was decreased. PC, SM, PE and PI profiles revealed mixed patterns with species overrepresented and underrepresented in apoA-Ideficient HDLs making up approximately equal shares.

\subsection{Bubble plots}

Similar to the heatmaps, the bubble plots revealed that abundances of numerous molecular species of LPC, PA and PG, as well as some Cer species were consistently increased in apoA-I-deficient HDLs (Fig. 3, Supplementary Figure S3). By contrast, PC, SM, PE and PI species revealed mixed patterns across HDL subpopulations, involving both elevated and reduced abundances. Importantly, a majority of lipid species significantly altered (FDR-corrected) by apoA-I deficiency were located in the middle-right part of the plots, indicating that they were moderately to highly abundant. This pattern was observed across all HDL subpopulations (Supplementary Figure S3). Together with the heatmaps, the bubble plots thereby demonstrated that the LPC, PA and PG species were altered most when compared to the control group, while being low abundant, whilst moderately- to highlyabundant species of PC and SM revealed mixed patterns.

\subsection{Structural analysis}

The next type of chart reveals the structure of lipid species significantly differing between the groups. Cornucopia of PC, LPC, PE, PA, and PG species with multiple double bonds in their fatty acid moieties showed significant 
differences (FDR-corrected) in their abundances between the patient and control groups, while differences in the species containing only saturated fatty acid residues did not reach significance (Fig. 4). There were several significantly different (FDR-corrected) between the groups fully saturated species of PC, LPC and Cer classes, while the majority of the species shown in Fig. 4, possessed one or several double bonds in their structure. The most prominent and consistent differences (FDR-corrected) were found throughout HDL subpopulations for PC 34:2 (which was significantly decreased in apoA-I-deficient vs control subjects), SM 32:1 and 34:0 (which were decreased), LPC 20:3 (which was increased), PA 34:1, 36:3, 36:4 and 38:4 (which were increased), and PG 34:1, 34:2, 36:1, 36:2 and 36:3 (which were increased), revealing that unsaturated molecular species were most strongly affected by the apoA-I deficiency in the HDL lipidome. Of particular interest was the PC 34:2 species, which, as mentioned above, not only was underrepresented in all five apoA-I deficient HDL subpopulations, but was also highly abundant, representing the major HDL phospholipid species ( $22 \mathrm{wt} \%$ of phospholipid+sphingolipid).

\subsection{Network analysis}

To elucidate the role of compositional alterations for biological properties of HDL particles, network analysis was performed for all correlations between functional metrics and individual lipids' abundances in HDLs, as well as abundances of apoA-I and apoA-II.

Compositional relationships revealed a systematic pattern across HDL subpopulations. Notably, SM, Cer and PS species formed three separate clusters, while LPC and PA species overlapped in another cluster. PC, PE and PI species were largely scattered around these clusters, with PG species on top (Fig 5A). SM species were located next to those of Cer, while PS species appeared in the vicinity of those of LPC, PA and PG.

As earlier reported by us, biological properties of HDL differed significantly between the control and patient groups for the majority of HDL subpopulations [12]. Indeed, cellular cholesterol efflux capacity was significantly reduced in the patient vs control group in all HDL subpopulations, except HDL3c. In addition, antioxidative activity of HDL, evaluated as oxidation rate in the propagation phase and maximal concentration of dienes at the end of the propagation phase, showed significant decrease in the patient group for the HDL3c subpopulation, 
although no differences were found in HDL3b [12]. By contrast, anti-apoptotic activitiy of HDL subpopulations evaluated in HUVEC exposed to starvation did not differ between apoA-I-deficient and control subjects (Supplementary Table S5). As reported by us earlier [12], inhibition of apoptosis by small, dense HDL3b was significantly higher than by large, light HDL2a in control subjects $(+48 \%, \mathrm{p}<0.05)$.

Cholesterol effflux capacity and antioxidative activity of HDLs were correlated with the abundances of multiple lipid species (Figs. 5B, 5C, 5D). Whereas cholesterol efflux capacity of HDLs was most closely associated with a single PC 34:2, as well as with numerous PS and PE species, the relationships between the HDL lipidome and antioxidative activity were less specific. Remarkably then, abundances of several species revealed significant correlations (FDR-corrected) with all the metrics of HDL functions assessed. The list of such species whose abundances correlated with both cholesterol efflux capacity and antioxidative metrics contained 6 PC, 2 SM, 1 PI, 4 PE, 6 PS, and 5 Cer species (Table 1). Curiously, this list contained only species with unsaturated fatty acid moieties. LPC, PA and PG species whose HDL content was markedly affected by apoA-I deficiency did not reveal significant associations with all the three functional metrics evaluated by us.

As expected, abundances of the species we observed to be positively correlated with the biological activities of HDL were significantly decreased (FDR-corrected) in apoA-I-deficient patients, while abundances of the species negatively correlated with those metrics were found to be increased (Table 1, green and red arrows).Abundances of 13 out of 24 species, linked to biological function of HDL did not show significant differences (FDR-corrected) between the groups in any HDL subpopulation. Strikingly, the abundance of PC 34:2 was decreased in apoA-Ideficient subjects throughout all five HDL subpopulations, while abundances of PC 40:4 and PC 40:8 differed in three HDL subpopulations. In addition, abundances of SM 42:1 and PS 38:4 differed in two HDL subpopulations, while abundances of PC 36:5, SM 41:1, PE 38:6, PE 40:7, Cer d18:2/14:0 and Cer d18:2/24:1 revealed differences in one HDL subpopulation only.

Finally, anti-apoptotic activity of HDL particles assessed as inhibition of annexin V binding to starving HUVEC cells was not significantly correlated with abundances of any lipid species (data not shown). Given that S1P may provide a key contribution to the capacity of HDL to reduce apoptosis [19], S1P content of HDL was evaluated to 
provide additional insight into compositional determinants of this biological activity. No difference in S1P content of HDL subpopulations was observed between apoA-I-deficient and control subjects (Supplementary Table S6). As reported by us earlier [17], S1P was more abundant in small, dense relative to large, light HDL in the both control and apoA-I-deficient groups. While no correlation between S1P abundance and biological activities of HDL was observed, there were significant (FDR-corrected) negative correlations of S1P with the abundances of Cer d18:1/18:0, Cer d18:1/19:0, Cer d18:1/24:0, Cer d18:1/25:0 and Cer d18:2/23:0 in HDL2a as well as of S1P with the abundances of PS 36:1 and PS 38:2 in HDL3a (data not shown).

\subsection{Metabolic pathways affected by apoA-I deficiency}

The list of lipid species whose abundances were correlated with the biological activities of HDL and were significantly differing between the groups was entered into the MetExplore web server [20] in order to identify metabolic pathways altered by apoA-I deficiency. The representation of the metabolic pathways identified was subsequently included in classical pathway representation (Fig. 6; a high-resolution version including names of intermediary metabolites is available as Supplementary Figure S4). The list of affected pathways included sphingolipid metabolism, glycerophospholipid metabolism, glycosylphosphatidylinositol-anchor biosynthesis, linoleic acid metabolism and alpha-linoleic acid metabolism (Supplementary Table S4). After BenjaminiHochberg correction, significant metabolic alterations were only detected for sphingolipid metabolism, glycerophospholipid metabolism and linoleic acid metabolism pathways.

\subsection{Lipid-related genes affected by apoA-I deficiency}

The list of lipid species whose abundances were correlated with the biological activities of HDL and were significantly differing between the groups (FDR-corrected) was further employed to identify lipid-related genes affected by apoA-I deficiency. For this purpose, the species names were converted to their corresponding Human Metabolome DataBase (HMDB) accessions and entered into the MetaboAnalyst Network Explorer tool. The analysis revealed seven genes being associated with impaired lipid metabolism in apoA-I deficiency, namely APOA1, LCAT, CDS1, CDS2, GLTP, PLA2G1B, and PCSK7 (Fig. 7). 


\section{Discussion}

In this study, HDL particles isolated from apoA-I-deficient patients revealed a markedly altered profile of molecular lipid species characterized by multiple perturbations in the phospho- and sphingolipidome, adding to the deficient biological activities of HDL. Importantly, the analysis of abundances of lipid species uncovered numerous alterations in apoA-I deficient HDLs, which remained hidden in the analysis of abundances of lipid classes performed by us earlier [12].

The perturbations of phospho- and sphingolipidome of HDL affected species from all lipid classes. Among highly-to-moderately abundant classes of PC, SM and PI, the pattern was complex, with some species featuring elevated abundances in apoA-I-deficient HDLs, while abundances of other species were reduced. Remarkably, apoA-I-deficient HDLs lacked numerous polyunsaturated lipid species, including PC 34:2 and PC 40:8, while possessing increased amounts of saturated species, including PC 32:0 and PC 34:0. It is generally accepted that saturated fatty acids exert proinflammatory actions, whereas the opposite can be said about polyunsaturated fatty acids which possess anti-inflammatory properties [25]. The imbalance of polyunsaturated relative to saturated species found in the present study may have contributed to elevated low-grade inflammation as observed by us in the apoA-I deficiency.

When plotted as a part of a correlation network map, PC, PE and PI species were scattered along parallel lines, ending in three clusters separately formed by SM, Cer and PS species, as well as in another cluster formed by LPC and PA species together. Such distribution can be attributed to LPC, PS and PA species predominantly present in small HDL3 particles, while SM and Cer species are known to be concentrated in large HDL2 [10]. Combining with functional characteristics of HDL, the network analysis demonstrated that multiple moderately to highly abundant PC and SM species were related to cholesterol efflux capacity and antioxidative activity of HDL. Unsurprisingly, abundances of the species that were decreased in apoA-I-deficient HDL subpopulations displayed positive correlations with the HDL functional metrics, while negative correlations were observed for the species 
whose HDL content was increased. Notwithstanding, all the species linked to functional properties of HDL carried one or more double bonds in their fatty acid moieties.

The clustering patterns revealed that cholesterol efflux capacity of HDL was located, at the network map, between the PS and PE clusters, in the vicinity of a single PC species of PC 34:2. The highly abundant PC 34:2, moderately abundant SM 42:1 and PC 40:4, as well as low abundant PC 40:8 species were all significantly associated with the both biological acitivities, and their HDL abundances were significantly different between the patient and control groups. Of particular interest, while SM lipid species revealed a number of links to biological functions of HDL, total SM was not correlated with HDL functional metrics in our previous study [12]. The associations with HDL function were thus masked by summarizing abundances of all the SM species in the previous study [12], emphasizing the strength of our present in-depth approach.

Major alterations were present in polyunsaturated lipid species of LPC, Cer, PA and PG species, manifesting themselves as their increased abundances in apoA-I-deficent vs. control HDL subpopulations. LPC is well-known to possess proinflammatory properties [26] and to display a high affinity for G-protein-coupled receptors, thereby playing a signaling role [27-29]. PA participates in the regulation of inflammation [30] and intracellular signaling [31] as well, while ceramides possess proapoptotic activity [32]. Although a whole array of LPC, Cer, PA and PG species was significantly enriched in apoA-I-deficient HDLs, the structure-function analysis revealed direct links with biological function of HDL only for Cer lipids. The species of the proatherogenic trio of LPC, PA and Cer may therefore have acted indirectly by promoting formation of a pro-inflammatory milieu, further deteriorating HDL function already hampered by the altered particle composition. In a similar fashion, PG species revealed significant between-group differences in their HDL content but their abundances were not associated with the HDL function.

Between-group differences in PE species were complex, with some apoA-I-deficient HDL subpopulations (HDL2b and 2a) displaying decreased PE content, while others (HDL3c) being enriched in PE. In particular, the content of a saturated species of PE 34:0 was significantly elevated in apoA-I-deficient HDL, adding up to the set of saturated species of PC 32:0 and PC 34:0 with increased HDL content described above. As in the correlation 
network map PE species were located closely to cholesterol efflux capacity of HDL, there were several PE species whose HDL content was correlated with the HDL function. Among them, species of PE 38:6 and PE 40:7 were significantly decreased in apoA-I-deficient HDL2b and 2a relative to control HDLs, consistent with their beneficial functional role.

Interestingly, altered biological functions of HDL in apoA-I-deficient subjects were confined to cholesterol efflux capacity and antioxidative activity and did not include anti-apoprotic properties. This result probably reflects the protein concentration basis employed for the measurements of anti-apoptotic activity of HDL. Indeed, apoA-Ideficient HDL were not deficient in S1P when the latter was expressed per unit protein, consistent with their normal capacity to reduce apoptosis.

We employed metabolic pathway ab intitio analysis to identify the pathways, possibly affected in the metabolism of apoA-I-deficient HDL. Our approach revealed that glycerophospholipid, sphingolipid and linoleic acid (LA) metabolism were significantly altered in apoA-I deficiency. Glycerophospholipid and sphingolipid metabolism are reportedly associated with atherosclerosis progression in apoE-deficient mice, with distinct plasma metabolomic profiles differentiating between the different stages of atherosclerotic progression [33]. Glycerophospholipids represent a common class of lipids critically important for integrity of cellular membranes; oxidation of esterified unsaturated fatty acid moieties dramatically alters biological activities of phospholipids [34]. ApoA-I-deficient HDL particles can be prone to oxidation due to increased amounts of pro-inflammatory lipids LPC and PA, as mentioned above. It is also worth noting that biological actions of phospholipids may potentially ameliorate the metabolic syndrome [35]. Finally, linoleic acid (LA) metabolism is tightly linked to atherogenesis. Indeed, it is well documented that LA exerts multiple anti-atherogenic activities. Conjugated LAs can reduce the concentration of atherogenic lipoproteins and the intensity of inflammatory processes in the plasma [36]. LA possess a protective effect against cholesterol accumulation in THP-1-derived macrophages [37]. Dietary supplementation of LA-rich fat, compared with a saturated fatty acid-rich fat in apolipoprotein E (apoE)-deficient mice, led to lowered atherosclerosis, reduced serum total cholesterol levels, increased HDL-cholesterol levels and lowered hepatic cholesterol levels [38]. 
Genes potentially playing a role in the observed alterations of the HDL lipidome and function in the apoA-Ideficiency, were identified using MetaboAnalyst's network analysis. Validating our approach, we found that the APOA1 gene was involved as it directly follows from the study design. This result is consistent with our earlier demonstration of perturbed HDL proteome in heterozygous apoA-I deficiency, primarily involving reduced content of apoA-I deficiency, equally that of apoA-II [12], which might both contribute to the defective HDL function. Other genes affected included lecithin-cholesterol acyltransferase (LCAT), CDP-diacylglycerol synthetase $1(C D S 1)$ and $2(C D S 2)$, glycolipid transfer protein (GLTP), group 1b phospholipase A2 (PLA2G1B), and proprotein convertase subtilisin/kexin type 7 (PCSK7). The presence of the LCAT gene in this list additionally supports our earlier observation of the dysregulation of LCAT in apoA-I deficiency [12]. Both CDS1 and CDS2 genes are known to regulate PI production [39], and thus, may contribute to the observed alterations of PI content. The GLTP gene is tightly linked to Cer biosynthesis [40], which was also disturbed in the apoA-I deficiency. The enzyme encoded by PLA2G1B gene is responsible for hydrolysis of PLs to LPC in the intestine [41], and may reflect increased LPC and decreased PC contents in the apoA-I deficiency. PCSK7 gene was reportedly associated with lipid metabolism [42], consistent with its association with the alterations found in the HDL lipidome in our study.

Expectedly, we observed that apoA-I and, as a consequence, HDL-C concentrations were significantly lower in the patient group than in controls, reflecting hampered apoA-I production in the patients [43]. In parallel, TG levels were significantly elevated in the patient group as much as one third from the control group level. Interestingly, this value can reach a much higher magnitude - available literature describes a case of a patient with apoA-I deficiency involving TG concentration as high as $417 \mathrm{mg} / \mathrm{dl}$, which is more that three times of what we observed in our study, despite intensive treatement with statins, ezetimibe, fenofibrate, and niacin [44]. These observations additionally emphasize the absence of efficient therapeutic strategy to treat this metabolic condition. Our present data may therefore have clinical implication. Indeed, they suggest that a proven therapy involving plasma transfusions in apoA-I-deficient patients [45-47] can be enhanced by HDL enriched in vitro with the lipids we found in this study to be positively associated with antiatherogenic function of HDL and to be reduced in 
apoA-I deficiency. The most obvious candidate for this approach represents such a major lipid of HDL as palmitoyllinoleoyl PC, a major isomer of PC 34:2.

In conclusion, our findings presented herein document multiple alterations in the molecular composition of HDL lipidome and their links to HDL functionality in heterozygous apoA-I deficiency involving a nonsense mutation at APOA1 codon -2. Multiplied by low circulating HDL concentrations, such deficiency in HDL composition and function can be expected to contribute to accelerated atherogenesis observed in this clinical condition. As a corollary, normalization of HDL phospho- and/or sphingolipid composition (i.e. via HDL enrichment in functional phospho- and/or sphingolipid through a diet or medical intervention), may represent promising therapeutic strategy to reduce cardiovascular risk in genetic apoA-I deficiency. 
These studies were supported by CAPES, FAPESP, National Institute for Health and Medical Research (INSERM) and Russian Science Foundation (Grant \# 18-15-00254). F.R. gratefully acknowledges financial support from the "Association pour la recherché sur Les Lipoproteines et l'Atherogenese" (ARLA, France).

Competing Interests

The authors who have taken part in this study declared that they do not have anything to disclose regarding funding or conflict of interest with respect to this manuscript.

Contribution Statement

EZ, FR, ML, MDA, PHB and PT performed experiments and production of data, MP and EZ performed calculations, CVSJ, RDS, MJC, AO and AK designed and directed the project. All authors have approved the final article.

\section{References}

1. Genest, J., Jr., et al., Familial hypoalphalipoproteinemia in premature coronary artery disease. Arterioscler Thromb, 1993. 13(12): p. 1728-37.

2. Genest, J.J., Jr., et al., Familial lipoprotein disorders in patients with premature coronary artery disease. Circulation, 1992. 85(6): p. 2025-33.

3. Schaefer, E.J., et al., Diagnosis and treatment of high density lipoprotein deficiency. Progress in Cardiovascular Diseases. 59(2): p. 97-106.

4. Schaefer, E.J., R.D. Santos, and B.F. Asztalos, Marked HDL deficiency and premature coronary heart disease. Curr Opin Lipidol, 2010. 21(4): p. 289-97.

5. Kontush, A., HDL-mediated mechanisms of protection in cardiovascular disease. Cardiovasc Res, 2014. 103(3): p. 341-9.

6. Fielding, C.J. and P.E. Fielding, Molecular physiology of reverse cholesterol transport. J Lipid Res, 1995. 36(2): p. 211-28.

7. latan, I., et al., Genetics of cholesterol efflux. Curr Atheroscler Rep, 2012. 14(3): p. 235-46.

8. Sorci-Thomas, M.G. and M.J. Thomas, The effects of altered apolipoprotein A-I structure on plasma HDL concentration. Trends Cardiovasc Med, 2002. 12(3): p. 121-8.

9. von Eckardstein, A., Differential diagnosis of familial high density lipoprotein deficiency syndromes. Atherosclerosis, 2006. 186(2): p. 231-9. 
10. Camont, L., et al., Small, dense high-density lipoprotein-3 particles are enriched in negatively charged phospholipids: relevance to cellular cholesterol efflux, antioxidative, antithrombotic, anti-inflammatory, and antiapoptotic functionalities. Arterioscler Thromb Vasc Biol, 2013. 33(12): p. 2715-23.

11. Kontush, A. and M.J. Chapman, Functionally defective high-density lipoprotein: a new therapeutic target at the crossroads of dyslipidemia, inflammation, and atherosclerosis. Pharmacol Rev, 2006. 58(3): p. 34274.

12. Rached, F., et al., Defective functionality of HDL particles in familial apoA-I deficiency: relevance of alterations in HDL lipidome and proteome. Journal of Lipid Research, 2014. 55(12): p. 2509-2520.

13. Santos, R.D., et al., Characterization of high density lipoprotein particles in familial apolipoprotein A-I deficiency. J Lipid Res, 2008. 49(2): p. 349-57.

14. Guérin, M., et al., Fenofibrate Reduces Plasma Cholesteryl Ester Transfer From HDL to VLDL and Normalizes the Atherogenic, Dense LDL Profile in Combined Hyperlipidemia. Arteriosclerosis, Thrombosis, and Vascular Biology, 1996. 16(6): p. 763-772.

15. Chapman, M.J., et al., A density gradient ultracentrifugal procedure for the isolation of the major lipoprotein classes from human serum. J Lipid Res, 1981. 22(2): p. 339-58.

16. Camont, L., M.J. Chapman, and A. Kontush, Biological activities of HDL subpopulations and their relevance to cardiovascular disease. Trends Mol Med, 2011. 17(10): p. 594-603.

17. Kontush, A., et al., Preferential sphingosine-1-phosphate enrichment and sphingomyelin depletion are key features of small dense HDL3 particles: relevance to antiapoptotic and antioxidative activities. Arterioscler Thromb Vasc Biol, 2007. 27(8): p. 1843-9.

18. Folch, J., M. Lees, and G.H. Sloane Stanley, A simple method for the isolation and purification of total lipides from animal tissues. J Biol Chem, 1957. 226(1): p. 497-509.

19. Nofer, J.-R., et al., Suppression of Endothelial Cell Apoptosis by High Density Lipoproteins (HDL) and HDLassociated Lysosphingolipids. Journal of Biological Chemistry, 2001. 276(37): p. 34480-34485.

20. Jourdan, F., et al., Use of reconstituted metabolic networks to assist in metabolomic data visualization and mining. Metabolomics, 2010. 6(2): p. 312-321.

21. Kanehisa, M., et al., Data, information, knowledge and principle: back to metabolism in KEGG. Nucleic Acids Res, 2014. 42(Database issue): p. D199-205.

22. Xia, J. and D.S. Wishart, Using MetaboAnalyst 3.0 for Comprehensive Metabolomics Data Analysis, in Current Protocols in Bioinformatics. 2002, John Wiley \& Sons, Inc.

23. Goeman, J.J., et al., A global test for groups of genes: testing association with a clinical outcome. Bioinformatics, 2004. 20(1): p. 93-9.

24. Hummel, M., R. Meister, and U. Mansmann, GlobalANCOVA: exploration and assessment of gene group effects. Bioinformatics, 2008. 24(1): p. 78-85.

25. Quehenberger, O. and E.A. Dennis, The human plasma lipidome. N Engl J Med, 2011. 365(19): p. 181223.

26. Marathe, G.K., et al., To hydrolyze or not to hydrolyze: the dilemma of platelet-activating factor acetylhydrolase. J Lipid Res, 2014. 55(9): p. 1847-54.

27. $\mathrm{Xu}, \mathrm{H}$. , et al., Targeted lipidomics - advances in profiling lysophosphocholine and platelet-activating factor second messengers. Febs j, 2013. 280(22): p. 5652-67.

28. Zmijewski, J.W., et al., Cell signalling by oxidized lipids and the role of reactive oxygen species in the endothelium. Biochem Soc Trans, 2005. 33(Pt 6): p. 1385-9.

29. Litvack, M.L. and N. Palaniyar, Review: Soluble innate immune pattern-recognition proteins for clearing dying cells and cellular components: implications on exacerbating or resolving inflammation. Innate Immun, 2010. 16(3): p. 191-200.

30. Lim, H.K., et al., Phosphatidic acid regulates systemic inflammatory responses by modulating the Aktmammalian target of rapamycin-p70 S6 kinase 1 pathway. J Biol Chem, 2003. 278(46): p. 45117-27. 
31. Delon, C., et al., Sphingosine kinase 1 is an intracellular effector of phosphatidic acid. J Biol Chem, 2004. 279(43): p. 44763-74.

32. Green, D.R., Apoptosis and sphingomyelin hydrolysis. The flip side. J Cell Biol, 2000. 150(1): p. F5-7.

33. Dang, V.T., et al., Comprehensive Plasma Metabolomic Analyses of Atherosclerotic Progression Reveal Alterations in Glycerophospholipid and Sphingolipid Metabolism in Apolipoprotein E-deficient Mice. Sci Rep, 2016. 6: p. 35037.

34. Bochkov, V.N., et al., Generation and biological activities of oxidized phospholipids. Antioxid Redox Signal, 2010. 12(8): p. 1009-59.

35. Castro-Gomez, P., et al., Relevance of dietary glycerophospholipids and sphingolipids to human health. Prostaglandins Leukot Essent Fatty Acids, 2015. 101: p. 41-51.

36. Stachowska, E., et al., Conjugated linoleic acid isomers may diminish human macrophages adhesion to endothelial surface. Int J Food Sci Nutr, 2012. 63(1): p. 30-5.

37. Song, Y., et al., Polyunsaturated fatty acid relatively decreases cholesterol content in THP-1 macrophagederived foam cell: partly correlates with expression profile of CIDE and PAT members. Lipids Health Dis, 2013. 12: p. 111.

38. Sato, M., et al., Linoleic acid-rich fats reduce atherosclerosis development beyond its oxidative and inflammatory stress-increasing effect in apolipoprotein E-deficient mice in comparison with saturated fatty acid-rich fats. Br J Nutr, 2005. 94(6): p. 896-901.

39. Lykidis, A., et al., The role of CDP-diacylglycerol synthetase and phosphatidylinositol synthase activity levels in the regulation of cellular phosphatidylinositol content. J Biol Chem, 1997. 272(52): p. 33402-9.

40. Malinina, L., et al., Sphingolipid transfer proteins defined by the GLTP-fold. Q Rev Biophys, 2015. 48(3): p. 281-322.

41. Hui, D.Y., Intestinal phospholipid and lysophospholipid metabolism in cardiometabolic disease. Curr Opin Lipidol, 2016. 27(5): p. 507-12.

42. Kurano, M., et al., Genome-wide association study of serum lipids confirms previously reported associations as well as new associations of common SNPs within PCSK7 gene with triglyceride. J Hum Genet, 2016. 61(5): p. 427-33.

43. Schaefer, E.J., P. Anthanont, and B.F. Asztalos, HDL metabolism, composition, function and deficiency. Current opinion in lipidology, 2014. 25(3): p. 194-199.

44. Lee, E.Y., et al., HDL Deficiency due to a New Insertion Mutation (ApoA-I(Nashua)) and Review of the Literature. Journal of clinical lipidology, 2013. 7(2): p. 169-173.

45. Kootte, R.S., et al., Effect of open-label infusion of an apoA-I-containing particle (CER-001) on RCT and artery wall thickness in patients with FHA. J Lipid Res, 2015. 56(3): p. 703-12.

46. Murayama, N., et al., Effects of plasma infusion on plasma lipids, apoproteins and plasma enzyme activities in familial lecithin: cholesterol acyltransferase deficiency. Eur J Clin Invest, 1984. 14(2): p. 122-9.

47. Shamburek, R.D., et al., Familial lecithin:cholesterol acyltransferase deficiency: First-in-human treatment with enzyme replacement. J Clin Lipidol, 2016. 10(2): p. 356-67. 


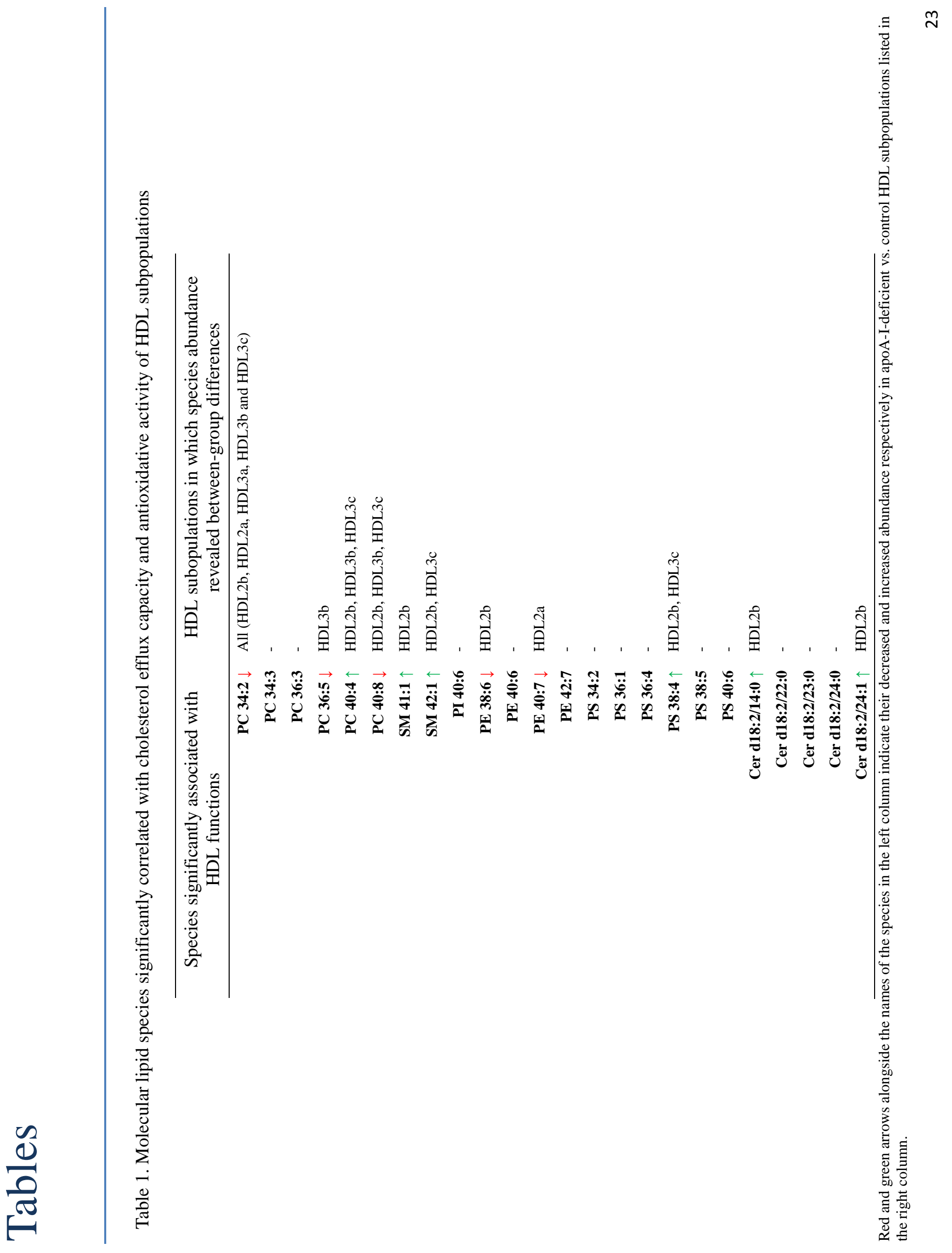




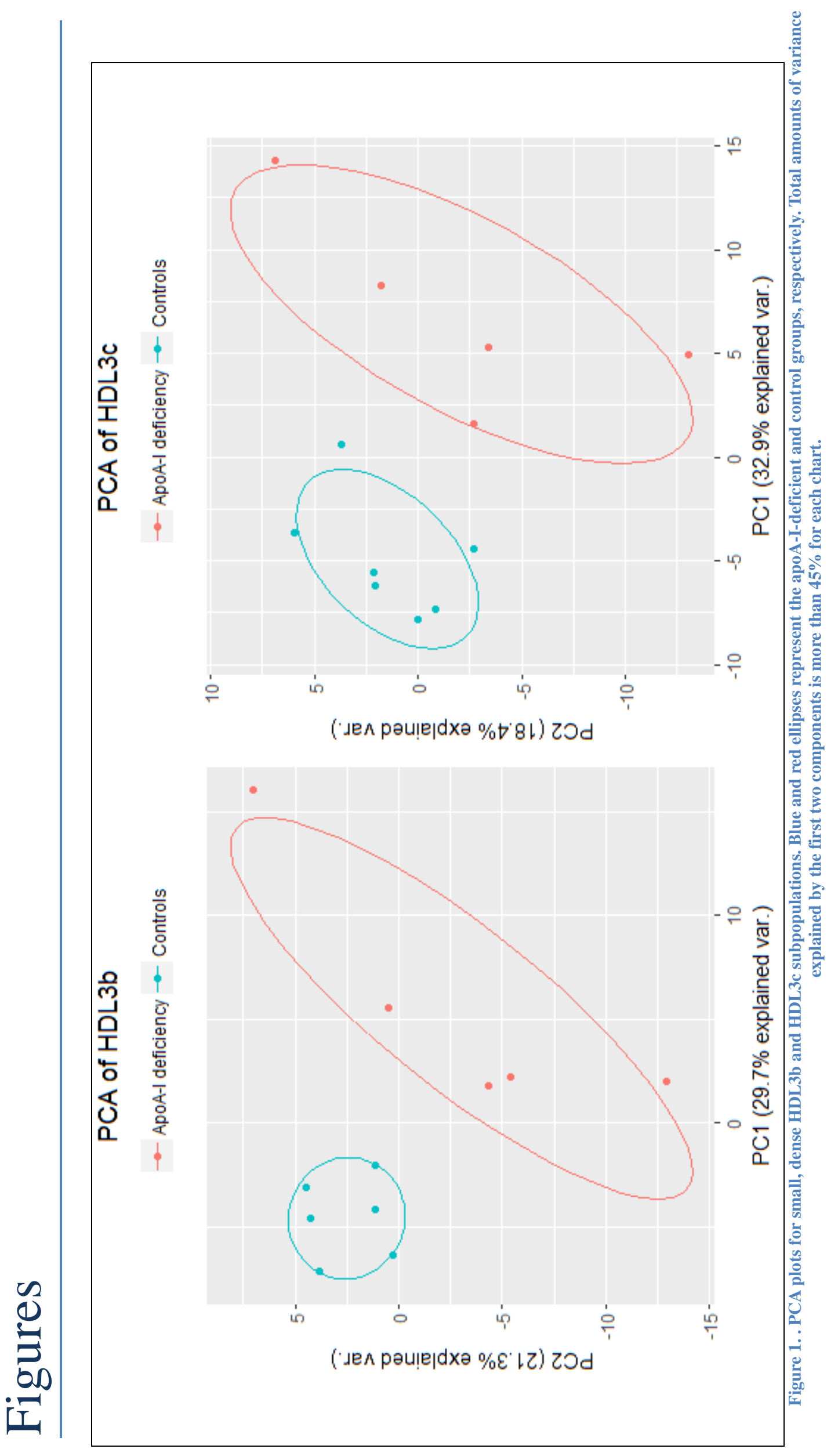




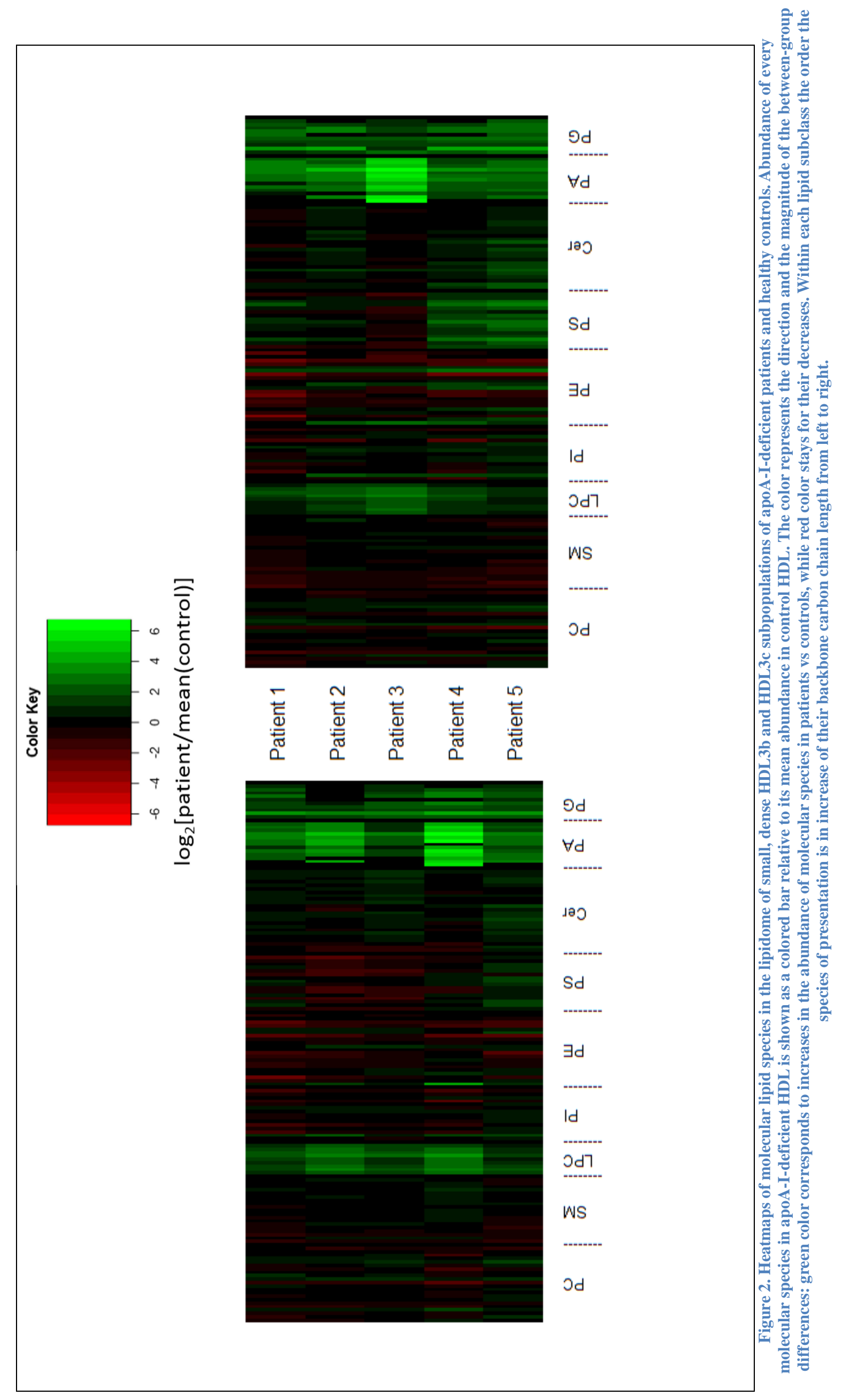




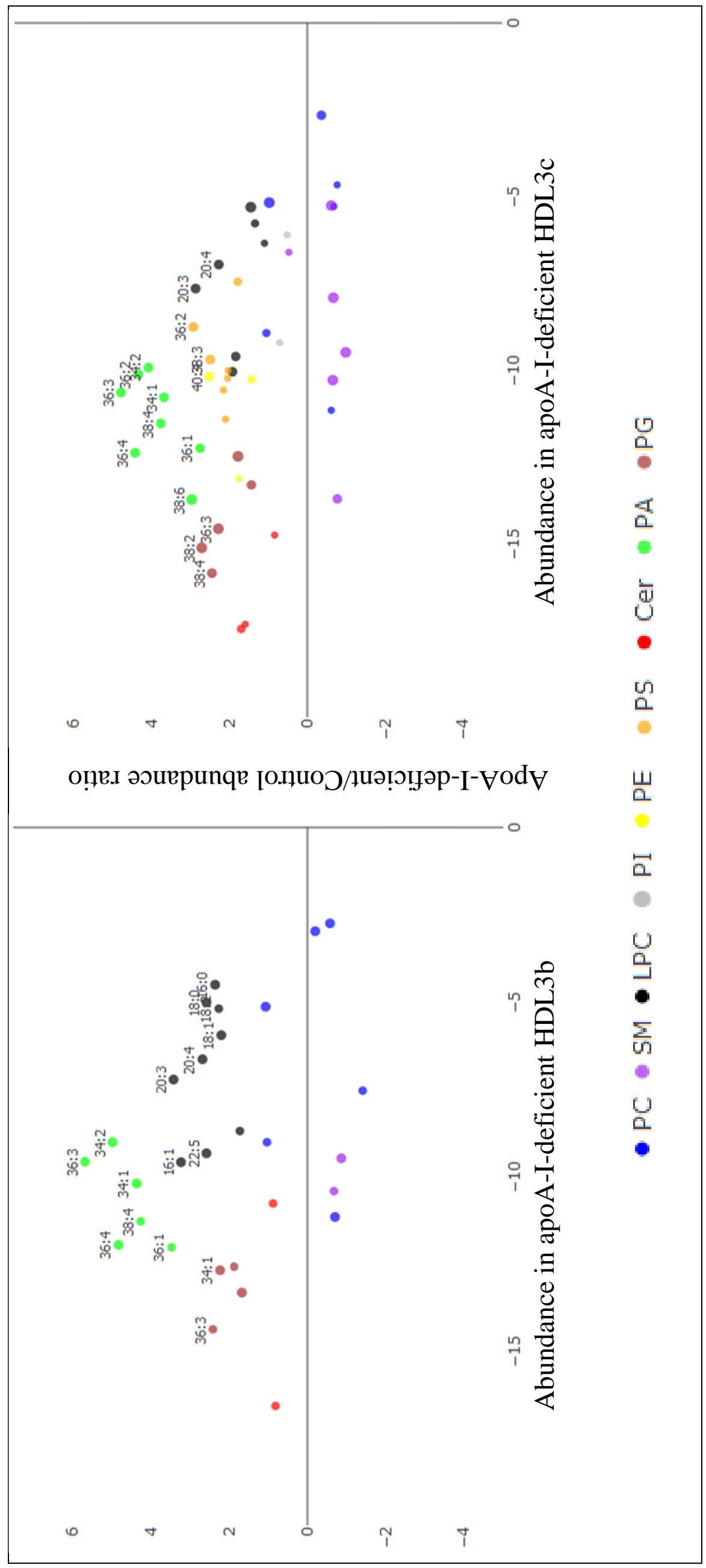

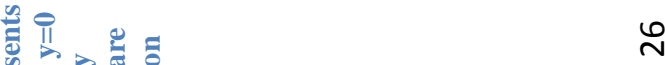

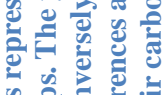

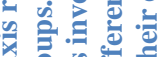

西 $\frac{100}{20}$

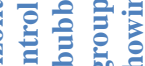

它语

원 है

.

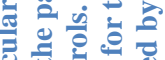

.

.

额议

芸

记

을

क्ष

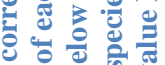

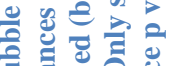

元

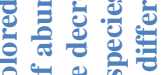

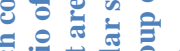

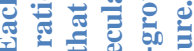

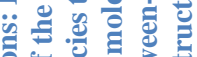

ㅎํ을

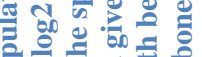

은

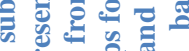

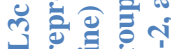

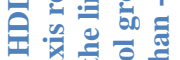

西

0.

政

角院

它

일

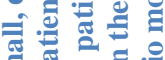

政哭

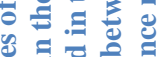

.

की

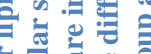

墕

列

㩆产

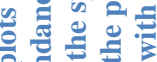

응

을

해을

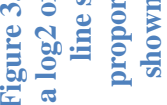



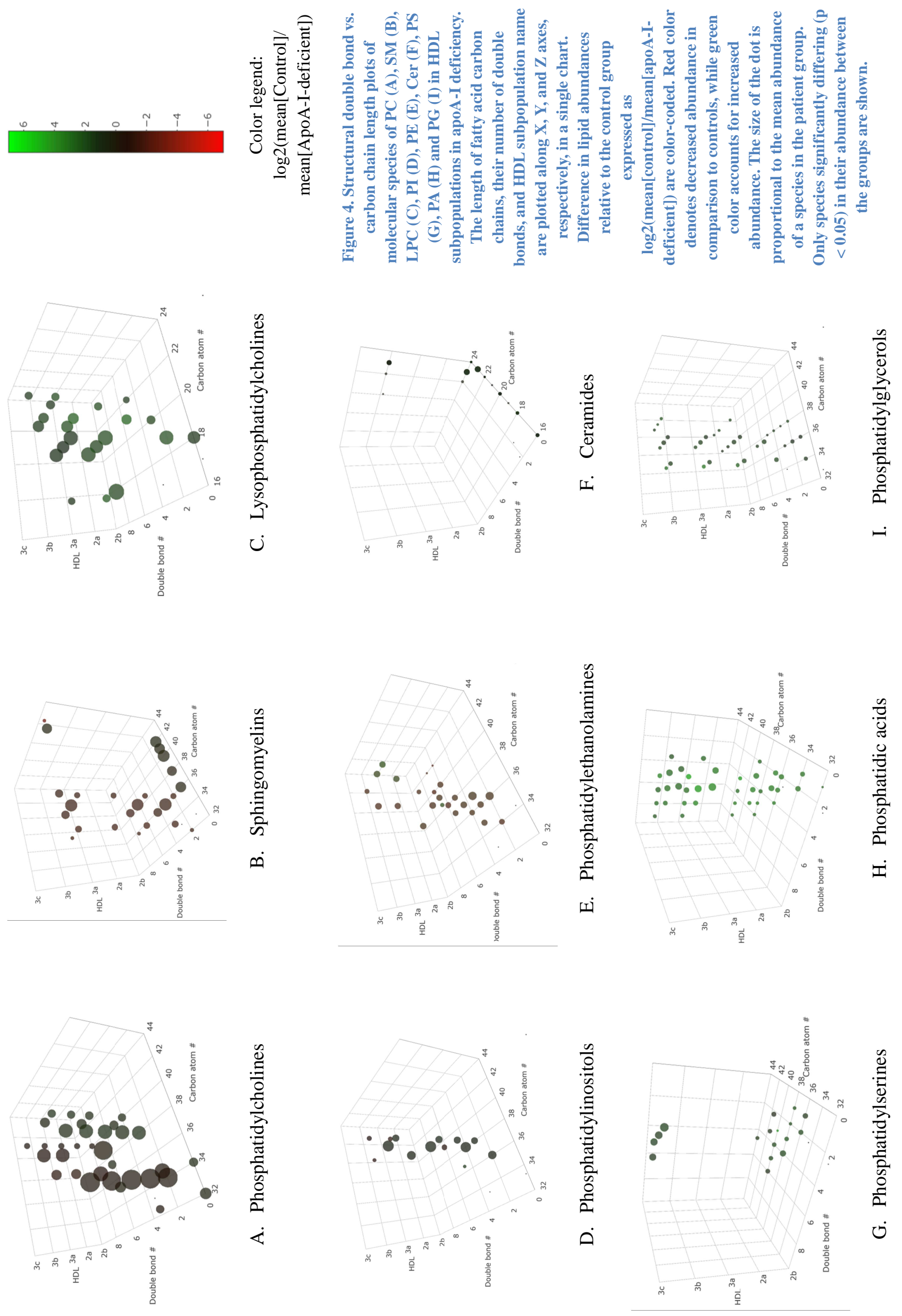


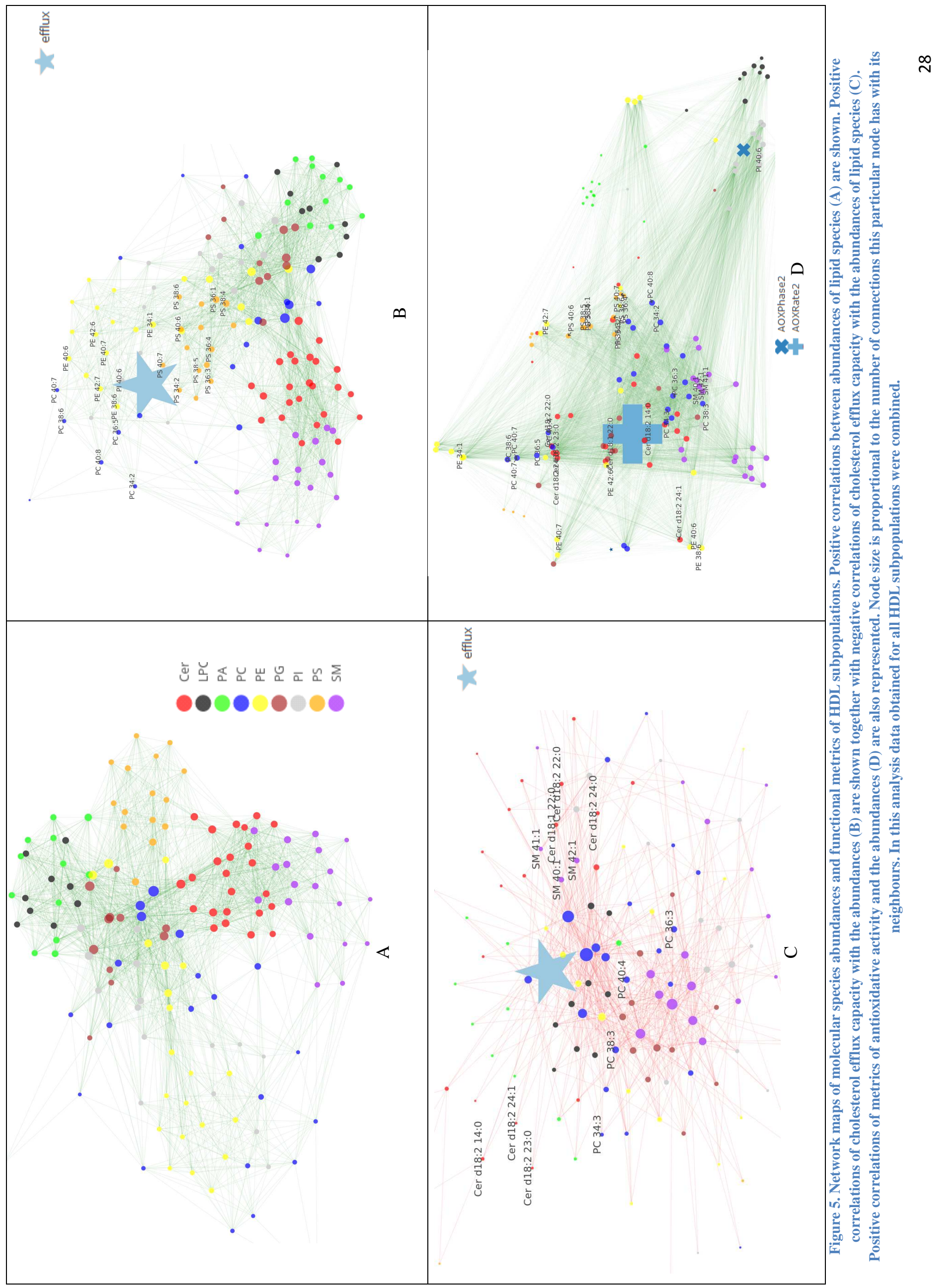



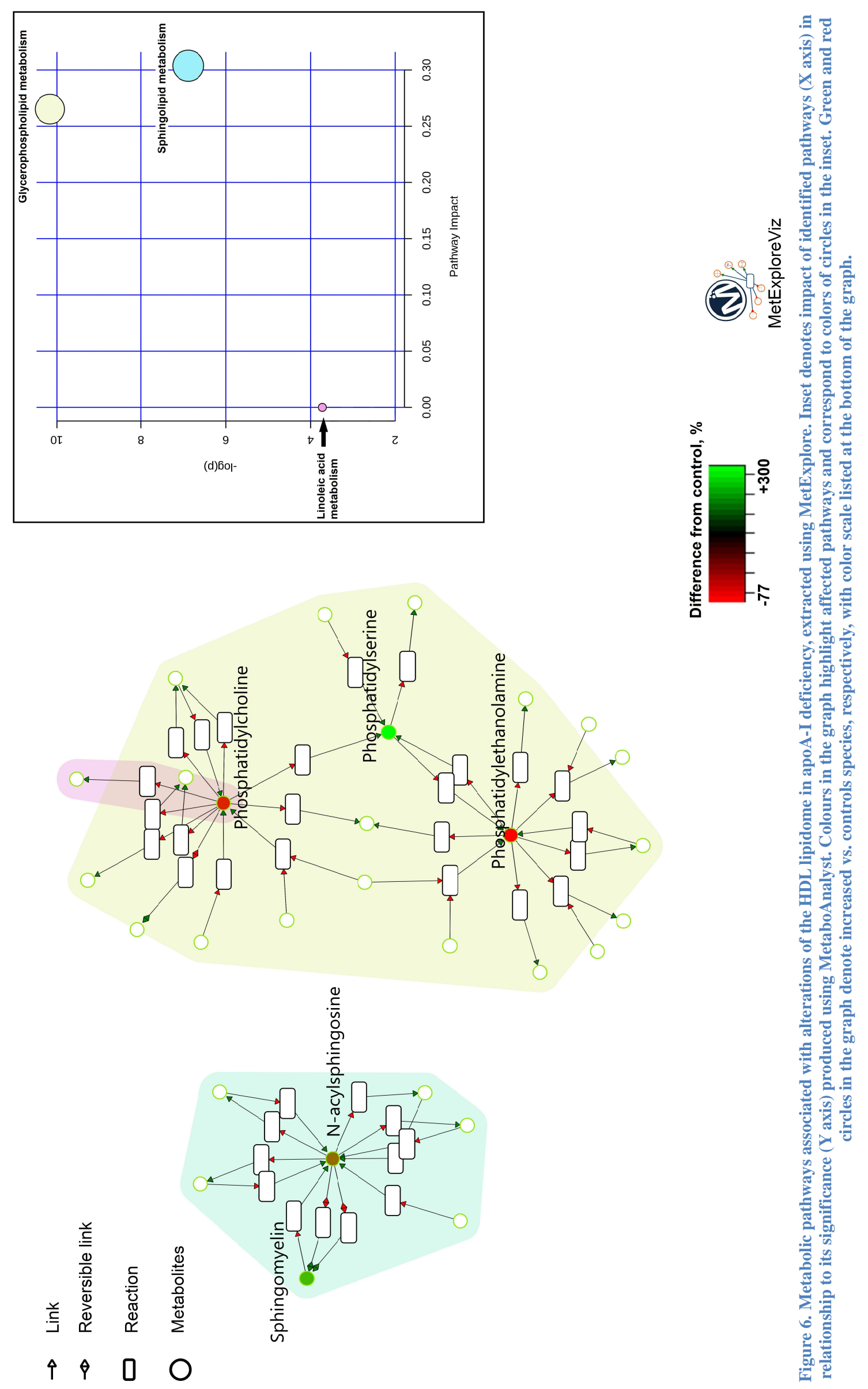

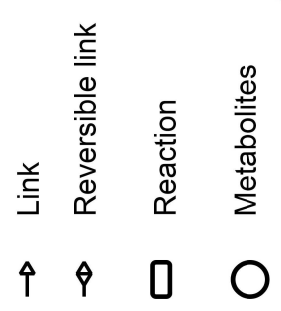

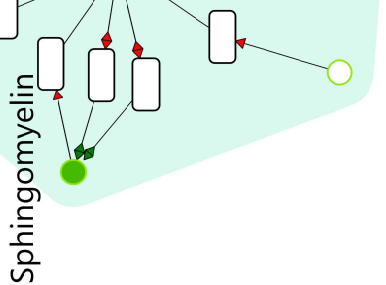




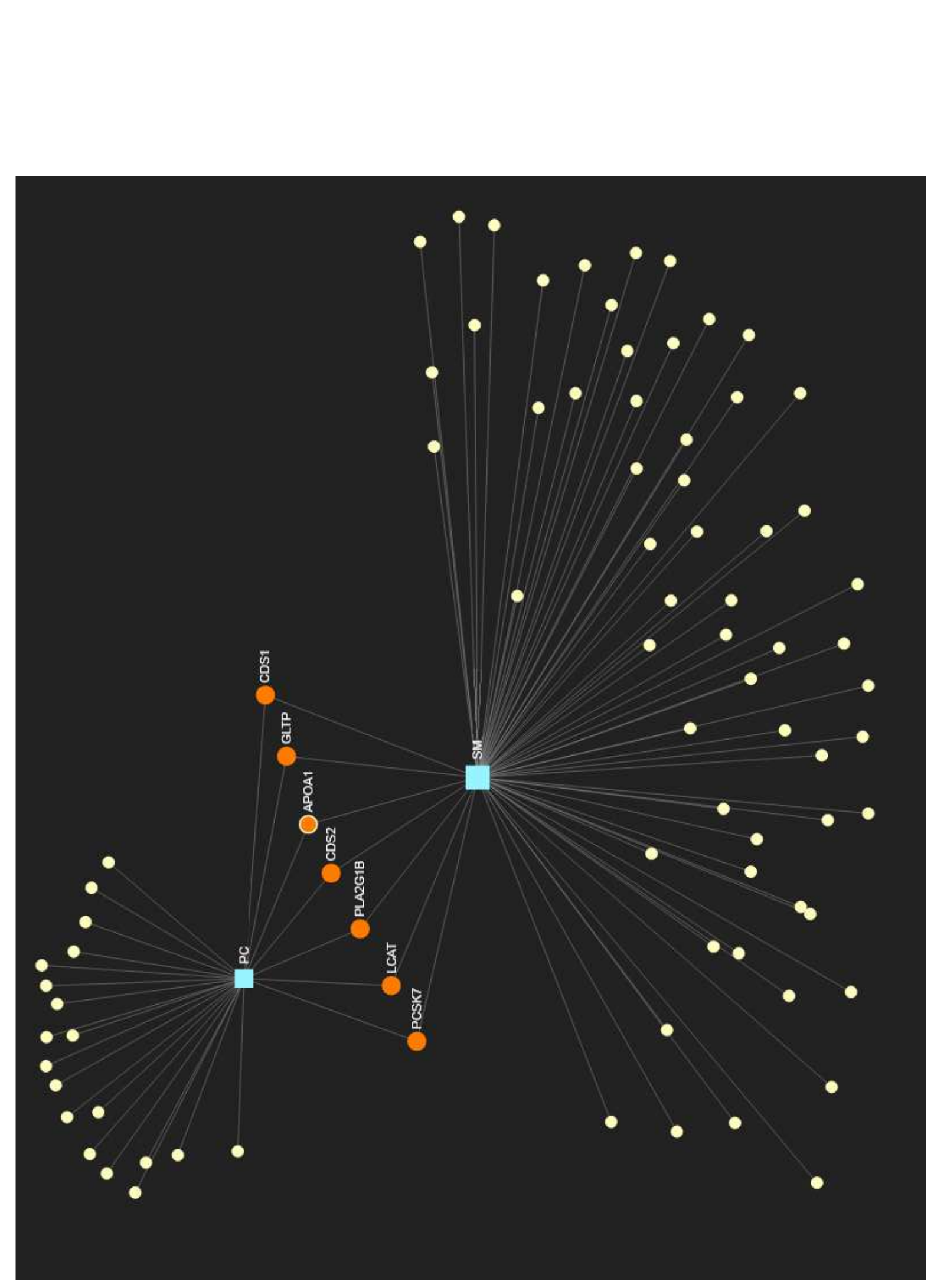

实



छิ

政

ํㅡㄹ

吾

की

运

政

플

告

을

플

क्षे

들

\%

局

ํㅡㄹ

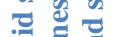

余

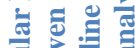

造

흘

를

훙

(5)

응

色

을

S.

흘

递

开

政

은 항

. $\equiv$ 㟧

政

흥

을

窟

施

可

结

定

대요

สำ

롱

要

可

需

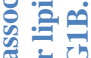

政

造

एँ

$\therefore$.

约

5

垔 